\title{
Alkaline phosphatase-positive cells isolated from human hearts have mesenchymal stem cell characteristics
}

\author{
Alessandra Melo de Aguiar $^{1^{*}}$, Crisciele Kuligovski ${ }^{1}$, \\ Marise Teresinha Brenner Affonso da Costa ${ }^{2}$, Marco Augusto Stimamiglio ${ }^{1}$, \\ Carmen Lúcia Kuniyoshi Rebelatto ${ }^{3}$, Alexandra Cristina Senegaglia ${ }^{3}$, \\ Paulo Roberto Slud Brofman ${ }^{3}$, Bruno Dallagiovanna ${ }^{1}$, Samuel Goldenberg ${ }^{1}$, \\ Alejandro Correa ${ }^{1}$ \\ ${ }^{1}$ Carlos Chagas Institute, Oswaldo Cruz Foundation, FIOCRUZ, Curitiba, Brazil; \\ *Corresponding Author: ale_aguiar@tecpar.br \\ ${ }^{2}$ Human Heart Valve Bank of Charity Hospital of the Brotherhood of Santa Casa de Misericordia de Curitiba, Curitiba, Brazil; \\ ${ }^{3}$ Core for Cell Technology, Pontifícia Universidade Católica do Paraná, Curitiba, Brazil.
}

Received 3 September 2011; revised 30 September 2011; accepted 11 October 2011.

\section{ABSTRACT}

Tissue-specific resident cells have been identified as a promising population of progenitor cells for cell-based therapies. We describe here the isolation from adult human hearts of tissue nonspecific alkaline phosphatase-positive cells (ALPL+ cells) with mesenchymal stem cell (MSC) characteristics. Samples from 24 adult cadaveric donors were obtained from a valve bank. Mean total ischemia time was $21.5 \pm 9.1$ hours. The success rate for the isolation of human heart-derived cells by the explant culture technique was $70 \%$ for the right auricle (14 of 20 trials) and 33\% for the right ventricle (7 of 21 trials). The total auricle-derived cell population (TAD) was used for the purification of ALPL+ cells. TAD and ALPL+ cells expressed markers for MSC and pericytes. TAD cells and ALPL+ cells differentiated into adipocytes, osteoblasts and chondroblasts, and ALPL+ cells expressed markers of these three lineages more strongly than TAD cells, as shown by RT-PCR. This population therefore has a greater potential for differentiation into mesechymal lineages than TAD cells. Both cell populations express some markers of cardiac progenitors, such as GATA4, CD117 and VEGF. ALPL+ cells expressed troponin $T$ and $A B C G 2$, which are also markers of the cardiac lineage. Heart samples from tissue banks could be considered as sources of MSC with putative commitment towards cardiac lineages, even after prolonged ischemia times.
Keywords: Alkaline Phosphatase; Mesenchymal Stem Cell; Pericyte; Heart; Cell Differentiation

\section{INTRODUCTION}

Mesenchymal stem cells (MSC) have been identified as a promising population of progenitor cells for cellbased therapies for the repair of mesenchymal tissue. Under appropriate conditions, they may give rise to various cell types with potential therapeutic applications, including osteoblasts, chondrocytes $[1,2]$ and cardiomyocytes [3-5]. Several sources of MSC have been identified, including bone marrow [6], adipose tissue [7], umbilical cord blood [8] and heart tissue [9,10].

MSC are characterized by the conditions required for their culture, their capacity to differentiate and a panel of various immunophenotyping markers, many of which are also expressed by fibroblasts and other cell types [11]. Several proteins have been identified as markers of the MSC population, for use alone or in combination with other markers. These proteins include mesenchymal stem cell antigen-1 (MSCA-1) [12]. This marker has recently been shown to be identical to tissue nonspecific alkaline phosphatase [13].

Alkaline phosphatase is a dimeric enzyme catalyzing the hydrolysis of phosphomonoesters, resulting in the release of inorganic phosphate from biomolecules [14]. This enzyme is present in all organisms and in many human tissues. Four isoforms have been described in humans: intestinal, placental, germ cell, and tissue nonspecific alkaline phosphatase [14]. Tissue nonspecific alkaline phosphatase is a phosphatidylinositol-linked plasma membrane glycoprotein, which can therefore be used as a cell marker for immunophenotype characteriza- 
tion and selection. This protein is a well known marker of embryonic stem cells (ESC) $[15,16]$. It is also a marker of neuron progenitor cells in mice [17]. Alkaline phosphatase-positive cells have also been identified as myogenic progenitor cells in human skeletal muscle, in which they were identified as pericytes [18]. Alkaline phosphatase has also been described as a perivascular marker present in MSC from the endometrium [19]. Indeed, the perivascular niche and the pericyte population have now been identified as possible MSC niches, as reviewed [20].

MSC can be isolated from the human heart, but it remains unclear whether the population of cells enriched in tissue nonspecific alkaline phosphatase has the same characteristics. In this study, we isolated and purified tissue nonspecific alkaline phosphatase-positive cells from heart tissue from cadaveric adult humans provided by a valve bank. We evaluated the characteristics of these cells as possible heart-derived MSC.

\section{MATERIALS AND METHODS}

\subsection{Collection of Human Cardiac Muscle Tissue}

Human cardiac muscle tissue was obtained from the human heart valve bank of Charity Hospital of the Brotherhood of Santa Casa de Misericordia de Curitiba, in accordance with the rules of the Oswaldo Cruz Foundation ethics committee (approval number 419/07). This investigation, carried out on human tissues, conformed to the principles outlined in the Helsinki Declaration. Tissue samples from the right auricle and/or right ventricle were collected from cadaveric donors were placed in F12 nutrient mixture supplemented with $2 \mathrm{mM}$ L-glutamine (GibcoTM Invitrogen Corporation, USA), 100 IU/ $\mathrm{ml}$ penicillin, and $0.1 \mathrm{mg} / \mathrm{ml}$ streptomycin (Sigma, USA). Tissue samples were maintained at $4^{\circ} \mathrm{C}$ for no longer than 48 hours before processing.

\subsection{Isolation and Culture of Human Heart-Derived Cells by Explant Culture}

Human heart-derived cells were isolated by explant culture. In brief, heart tissues were rinsed in HBSS (Hanks balanced salt solution) and their mass was determined. We then used scalpels to dissect 500 to $900 \mathrm{mg}$ of tissue into fragments $1-2 \mathrm{~mm}^{3}$ in size.

We cultured about $100 \mathrm{mg}$ of tissue fragments in each $25 \mathrm{~cm}^{2}$ culture flask (TPP, Switzerland). The tissue fragments adhered to the culture flasks coated with collagen film (Sigma, USA) after incubation at $37^{\circ} \mathrm{C}$ for one hour, in a humidified atmosphere containing $5 \%$ carbon dioxide. We then added culture medium. The composition of the maintenance medium was similar to that previously described [18], consisting of MegaCell ${ }^{\mathrm{TM}}$ DMEM (Sigma, USA) supplemented with 5\% fetal bovine serum, 2 mM L-glutamine (GibcoTM Invitrogen Corporation, USA), $5 \mathrm{ng} / \mathrm{ml}$ basic fibroblast growth factor, $0.1 \mathrm{mM} \beta$ mercaptoethanol, $1 \%$ non essential amino acids, 100 $\mathrm{IU} / \mathrm{ml}$ penicillin and $0.1 \mathrm{mg} / \mathrm{ml}$ streptomycin (Sigma, USA).

The tissue fragments were cultured for 15 to 30 days, with the replacement of $50 \%$ of the medium weekly. After the cells had migrated from the explants, they were harvested by digestion with $0.025 \%$ trypsin (GibcoTM Invitrogen Corporation, USA) in $0.02 \%$ EDTA (Sigma, USA). Tissue fragments were separated from the cell suspension by passage through a cell strainer with a 40 $\mu \mathrm{m}$ mesh (BD FalconTM, USA). Cells were plated at a density of $0.2-0.5 \times 10^{4}$ cells $/ \mathrm{cm}^{2}$. Cultured cells from passages 2 to 6 were used for all experiments.

\subsection{Purification of Alkaline Phosphatase-Positive Cells}

For the selection of tissue nonspecific alkaline phosphatase-positive cells (ALPL+), the total auricle-derived cell population (TAD) was incubated with a biotinylated antibody directed against the human tissue nonspecific alkaline phosphatase (R\&D Systems, USA). ALPL+ cells were purified with the CellectionTM anti mouse IgG kit (Invitrogen Dynal AS, Norway), in accordance with the manufacturer's instructions. Purified cells were cultured in maintenance medium and subjected to immunophenotyping, gene expression analysis and differentiation assays.

\subsection{Immunophenotyping by Flow Cytometry}

Flow cytometry data were acquired and analyzed as previously described [2]. Briefly, $2 \times 10^{5}$ human heartderived cells from passages 2 to 6 were labeled with antibodies against the following human proteins: CD90, CD14, HLA-DR, CD73 and CD140b (BD Pharmingen, San Jose, CA, USA) CD31, CD45, CD34 and CD133/1 (Miltenyi Biotec, Germany), CD105 and CD117 (E-bioscience, USA), tissue nonspecific alkaline phosphatase (R \&D Systems, USA), and nestin (BD Pharmingen, USA). For intracellular staining, cells were permeabilized with Fix\&Perm reagent (Caltag Laboratories, USA), in accordance with the manufacturer's instructions. Mouse isotype IgG1 antibodies were used as controls (BD PHARMINGENTM, USA). About 10,000 labeled cells were acquired with a FACSCalibur flow cytometer (Becton Dickinson, USA) and analyzed with FlowJo software (Flowjo, USA). 


\subsection{Differentiation into Mesenchymal Lineages}

TAD and ALPL+ cells were evaluated by inducing their differentiation into adipocytes, osteoblasts and chondroblasts, as previously described [2]. Cells between passages 2 and 6 were incubated with control maintenance medium or differentiation induction medium for 21 days. Cells were then fixed for morphological evaluation by standard staining procedures, with Oil Red for adipogenesis, Von Kossa staining for osteogenesis and toluidine blue staining for chondrogenesis [2]. We also performed RT-qPCR to estimate the level of differentiation-specific mRNA in induced TAD and ALPL+ cells. We analyzed adipogenic differentiation quantitatively, by counting cells from three biological replicates of TAD and ALPL+ cells, with analysis by ImageJ version $1.45 \mathrm{~d}$.

\subsection{Evaluation of Gene Expression by RT-qPCR}

Total RNA was obtained with the RNeasy kit (QIA GEN, USA) and samples were treated "in column" with DNAse I (QIAGEN, USA), in accordance with the manufacturer's instructions. Complementary DNA (cDNA) was synthesized from $1 \mu \mathrm{g}$ of total RNA, with oligo-dT primers (USB Corporation, USA) and a reverse transcriptase kit (IMPROM II, Promega, USA), according to the manufacturers' instructions. PCR was carried out as previously described $[2,25,23]$ The primer sets and PCR conditions are listed in Table 1.

\subsection{Statistical Analysis}

Donor age and total ischemia time are expressed as means \pm standard deviation. All other data are expressed as means \pm standard error for three or more biological replicates. We used Fisher Exact Test to compare successful cell isolations from auricle or ventricle samples. We used Student's t-test to compare other samples ( $\mathrm{p}<$ 0.05 considered significant).

\section{RESULTS}

\subsection{Isolation of Human Heart-Derived Cells by Explant Cell Culture}

We used the explant cell culture technique to isolate human heart-derived cells from right ventricles or auricles. Samples were obtained from 24 adult cadaveric donors

Table 1. Primer sets.

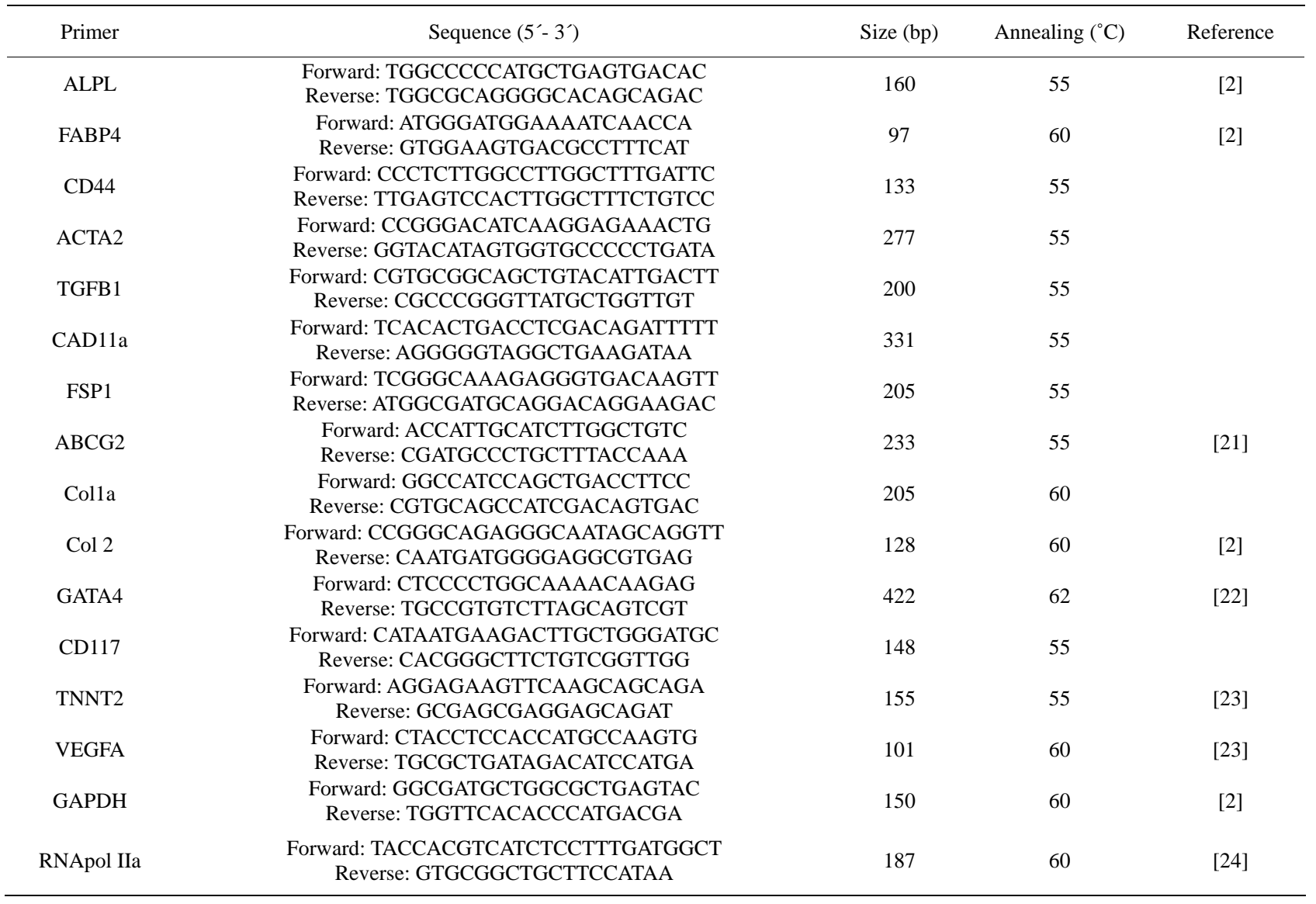


with a mean total ischemia time of $21.5 \pm 9.1$ hours (range: 2.5 to 41.8 hours). We have not found significant statistical difference when comparing ischemia time and successful and not successful cell isolation (data not shown). The mean age of the donors was 39 years \pm 12 ; $54 \%(n=13)$ of the donors were male and $46 \%(n=11)$ were female. After two to four weeks of culture, cells began to migrate from the cultured explants of auricle and ventricle tissues (Figures 1(a)-(b)).

Cells were successfully isolated in 14/20 trials for the right auricle (70\%) and 7/21 trials for the right ventricle (33\%) with a statistical significance of $p<0.05$. Success rates were similar for cultures of explants from male and female donors. However, we did not find statistical significance when comparing isolation success rates from auricle or ventricle samples and donor age classes. We observed a tendency of successful cell isolation in right ventricle samples from younger donors (Figure 1(c)). Cell isolation success rate from right ventricle samples was $50 \%$ for younger donors, but only $17 \%$ for older donors. Interestingly, samples from donors between the ages of 31 and 50 gave the highest success rates for cells isolation from right auricle samples. We obtained success rates of $86 \%$ for donors aged 31 to 40 years and $100 \%$ for donors aged 41 to 50 years. By contrast, success rates were lower $(50 \%)$ for auricle samples from donors between the ages of 21 and 30 years or between the ages of 51 and 60 years (Figure 1(c)). In addition, for 17 donors, we evaluated the isolation of human heart-derived cells from both the right ventricle and the right auricle. The success rates for isolation were different for the two explants. We were able to establish cell cultures from 12/17 (70\%) auricles and 4/17 (23\%) ventricles. Thus, the type of tissue used seems to play an important role in determining the success of human heart-derived cell isolation. These data suggest that the total auricle-derived cell population (TAD) may be a more suitable source of heart-derived cells than the population of cells from the right ventricle.

\subsection{Auricle- and Ventricle-Derived Cell Cultures are Heterogeneous and Express MSC Markers}

We characterized total auricle-derived and total ventricle-derived cell populations by performing RT-PCR and flow cytometry assays. MSC/pericyte markers, such as ALPL, CD44 and alpha smoth muscle actin (ACTA2), were detected in the cell samples by RT-PCR (Figure 2(a)). We also found markers of myofibroblasts, such as transforming growth factor beta 1 (TGF $\beta 1$ ) and cadherin 11a (Cad11a), and markers for fibroblasts, such as collagen 1a (Col1A) and fibroblast-specific protein 1 (FSP1) (Figure 2(a)). Thus, cell populations derived from auricle

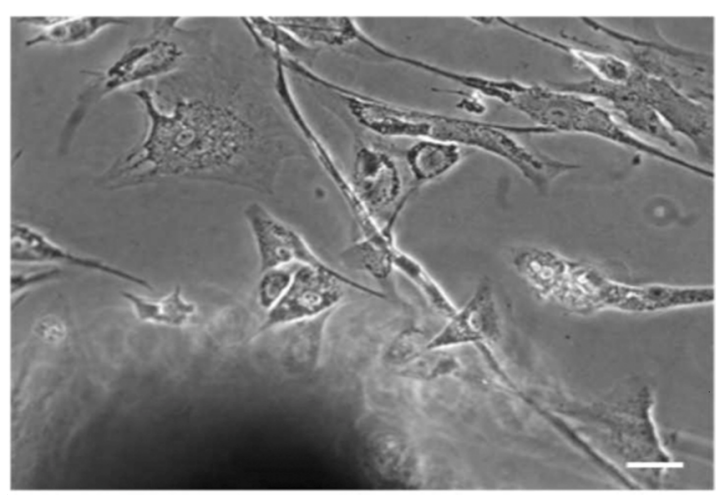

(a)

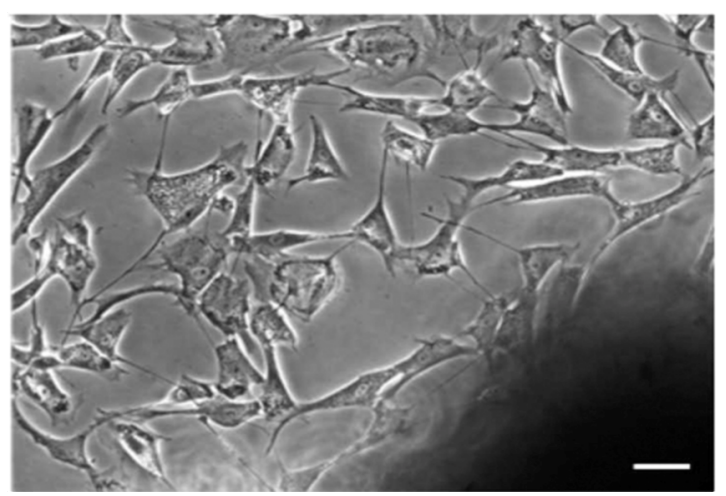

(b)

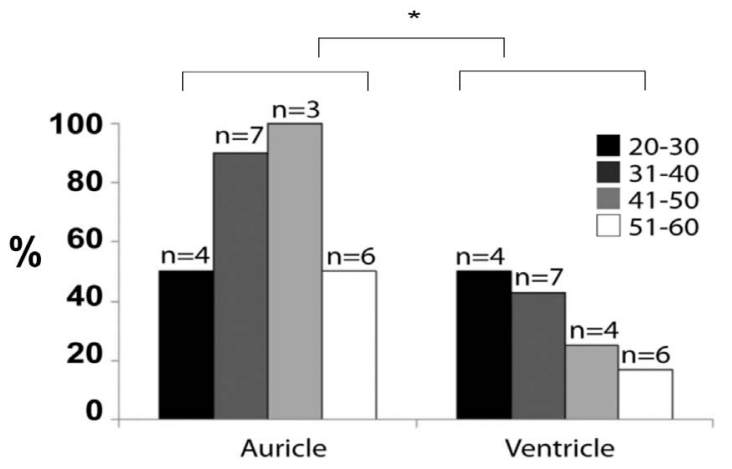

(c)

Figure 1. Isolation of cells derived from human heart. Contrast phase microscopy images, showing cells that have migrated from the right auricle (a) and right ventricle (b) after 2 to 4 weeks of primary explant culture. The success rates for the isolation procedure are shown as a function of tissue and donor age (c). Bars indicate age classes. The $y$ axis shows the percentage of successful isolations from auricle or ventricle cardiac muscle for each age class. The $n$ for each age class is indicated at the top of each bar. The calibration bar corresponds to 30 $\mu \mathrm{m} .{ }^{*} \mathrm{p}<0.05$.

or ventricle explant cultures were heterogeneous and RT-PCR found no difference in the profile of marker expression between them.

However, auricle-derived cell population had higher 
levels of CD90 and ALPL expression than ventriclederived cells on flow cytometry, whereas amounts of CD105, CD140b and CD117 were similar in both populations (Figure 2(b)). The auricle-derived cell population may be a more suitable source of heart-derived cells expressing tissue nonspecific alkaline phosphatase than the right ventricle as we found larger amounts of ALPL in auricle samples than in ventricle samples. We therefore used total auricle-derived cells (TAD) for subsequent analyses.

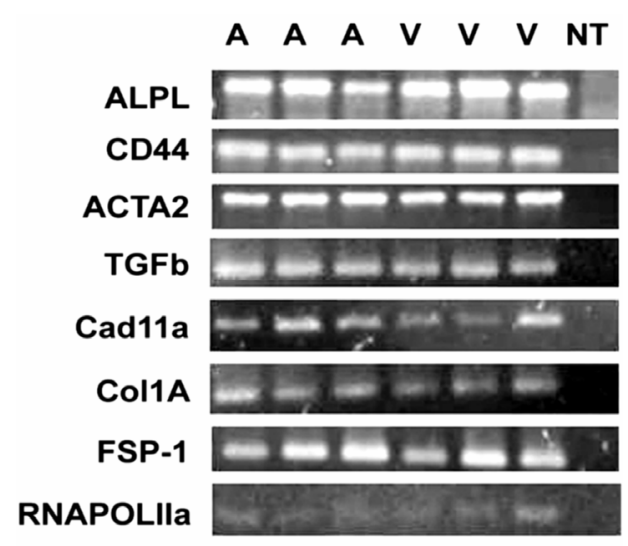

(a)

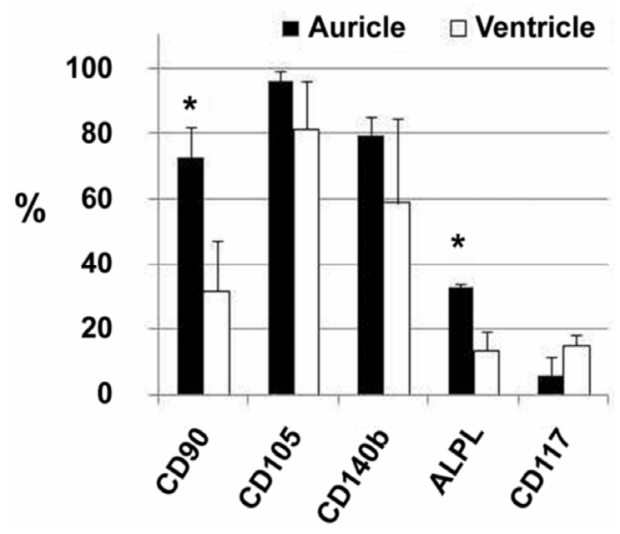

(b)

Figure 2. Characterization of auricle-derived and ventricle-derived cell cultures. The expression of markers for MSC/pericytes (ALPL, CD44, ACTA2), myofibroblasts (TGF-, Cad11a) and fibroblasts (FSP-1, col1a) and the expression of the housekeeping gene RNA Pol IIa were assayed by RT-PCR, with three independent samples at passage 4 . Samples from auriclederived cell cultures (A) and ventricle-derived cell cultures (V) are shown, NT (non template control) (a) The presence of markers for pericytes (ALPL, CD140b), mesenchymal stem cells (CD90 and CD105) and cardiac progenitor cells (CD117) was analyzed by flow cytometry (b) At least three independent donors were used for each marker. ${ }^{*} \mathrm{p}<0.05$.

\subsection{The ALPL+ Cell Population Can be Purified from Total Auricle-Derived cells (TAD) and Has the Characteristics and Differentiation Potential of MSC}

Using magnetic microbeads, we obtained a cell population enriched in cells expressing tissue nonspecific alkaline phosphatase (ALPL+). A single round of purifycation yielded an ALPL+ enriched population of 86.4\% $\pm 6.2 \%(\mathrm{n}=3)$, as quantified by flow cytometry (Figure 3(a)). We then evaluated markers of MSC, pericytes and endothelium cells. We observed no difference in the expression of MSC/perivascular cell markers, such as CD 140b and nestin (Figure 3(b)).

We analyzed the expression of endothelial markers: CD34 was expressed by less than 15\% of cells, Von Willebrand factor (vWF) was expressed by less than $10 \%$ of cells and other endothelial markers, such as CD133 and CD31, were not detected at all (Figure 3(c)). We then evaluated MSC markers. Both TAD and ALPL+ cells expressed positive markers for mesenchymal stem cells, such as CD90, CD105 and CD73 (Figure 3(d)), and neither of these cell populations expressed negative markers, such as CD45, CD14 and HLA-DR (Figure 3(e)). Thus, these heart-derived cell populations appear to contain MSC-like cells in accordance with criteria pointed out by Dominici et al. [20].

Thus, the differentiation of TAD and ALPL+ cells into osteoblasts, adipocytes and chondroblasts was induced. Both populations were able to differentiate along these three lineages, indicating that MSC-like cells are present in both TAD and ALPL+ cells, as shown by phase-contrast microscopy and cytochemistry (Figures 4 (a)-(b)). The expression of fabp4, collagen 1a and collagen II, lineage-specific markers of adipocytes, osteoblasts and chondroblasts, respectively, was assayed by RT-qPCR (Figure 4(c)). Fabp4, collagen 1a and collagen II mRNA levels were higher in ALPL+ cultures than in TAD cultures $(\mathrm{p}<0.05)$.

We analyzed adipogenic differentiation, by staining lipid-rich vacuoles with Oil Red O. We observed such differentiation in $48.5 \% \pm 16.1 \%$ of TAD cells and $69.6 \%$ $\pm 5.5 \%$ of ALPL + cells, this difference being non signifycant. TAD cells had only a few, very small intracellular lipid droplets (Figure 4(b)), whereas ALPL+ cells were large and round, with lipid-rich vacuoles in the cytoplasm (Figure 4(b)). We also evaluated the area, in pixels, covered by lipid-rich vacuoles stained with Oil Red $\mathrm{O}$ within an arbitrarily defined region, for three independent donors. The area covered by lipid-rich vacuoles was significantly larger for ALPL+ cells than for TAD (Figure 5). Thus, the adipocytes differentiated in ALPL+ cells were more mature than those in TAD cultures. 


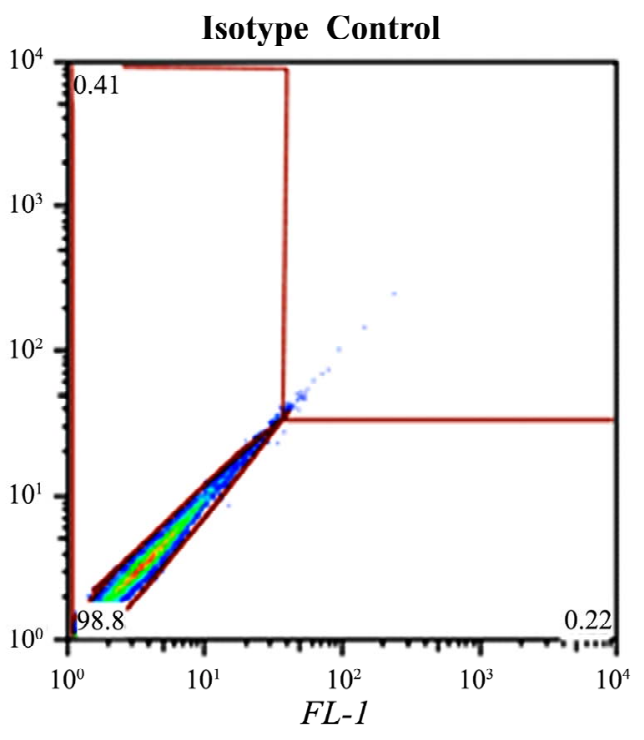

TAD
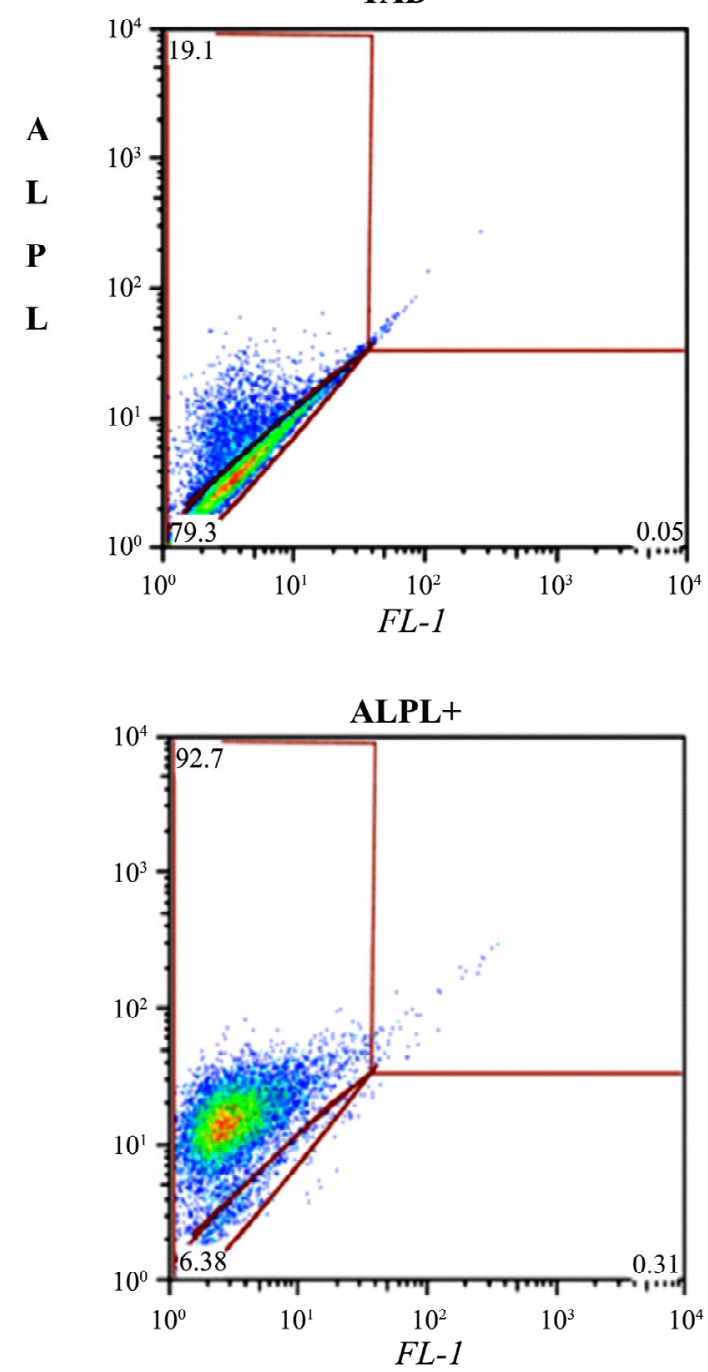

(a)

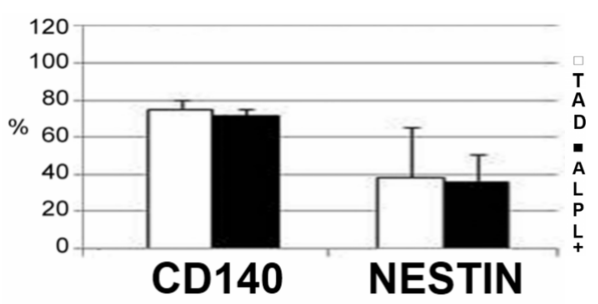

(b)

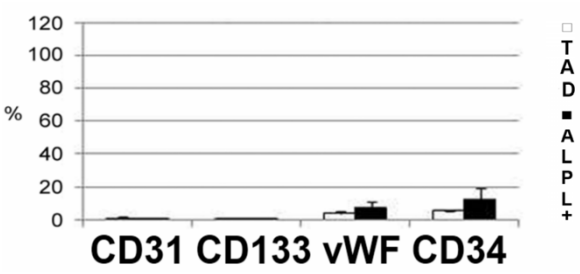

(c)

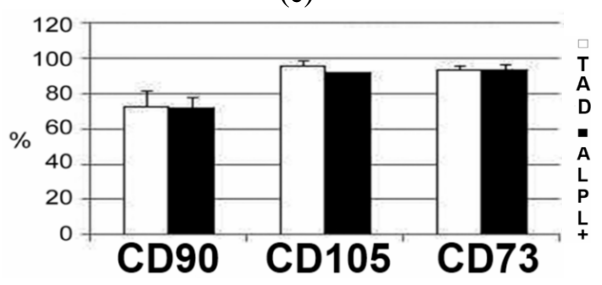

(d)

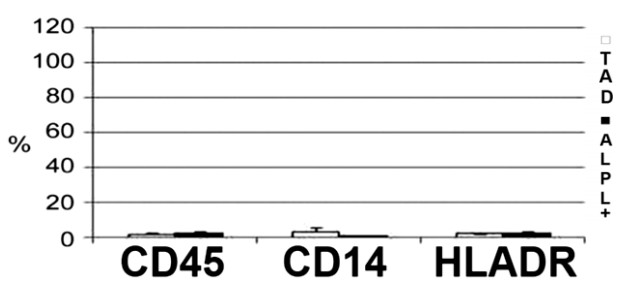

(e)

Figure 3. Purification of the ALPL+ population from auricle-derived cell cultures. Isotype control, TAD cells (and the ALPL+-enriched population after selection on micromagnetic beads and evalution by flow cytometry, a representative experiment (a) Markers for pericytes (b) Markers for endothelium (c) MSC-positive Markers (d) and MSC-negative markers (e) Imunnofenotyping was evaluated in TAD (total auricle-derived cell population), white bars and in ALPL+ cells (tissue nonspecific alkaline phosphatase positive cell population), black bars. Data are expressed as medians and standard error $(\mathrm{n}=3)$. Von Willebrand factor $(\mathrm{vWF})$.

\subsection{Expression of Cardiac Markers}

We evaluated the expression of cardiac markers (Figure 6). Both the TAD and ALPL+ cell populations expressed GATA4, an early cardiomyogenic marker [26], and CD117, a cardiac progenitor marker [27]. We also observed the expression of VEGF, an endothelial growth factor [28]. ALPL+ cells also expressed another marker of cardiac-resident stem cells: the side population marker ABCG2 [29,30]. In addition, these cells expressed cardiac troponin $\mathrm{T}$, another cardiac lineage marker. 


\section{DISCUSSION}

The aim of this study was to isolate ALPL+ cells from human heart samples from a valve bank. We showed that a heart-derived cell population enriched in cells expressing tissue nonspecific alkaline phosphatase had mesenchymal stem cells characteristics such as plastic adherence, antigenic profile and the potential to differentiate into mesenchymal lineages [20]. We also showed that heart-derived ALPL+ cells expressed a repertoire of cardiac markers.

Alkaline phosphatase has been shown to be a marker for MSC in bone marrow, embryonic stem cells, neuronal progenitor cells, myogenic pericytes in human skeletal muscle and endometrial MSC-like cells [12,13,15, 17-19]. We investigated ALPL+ cells in human hearts.

In most previous studies, the cardiac progenitor cells

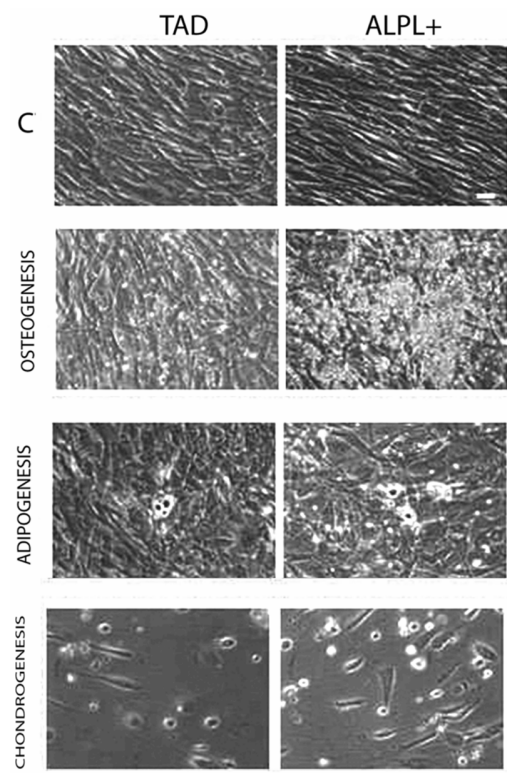

(a)
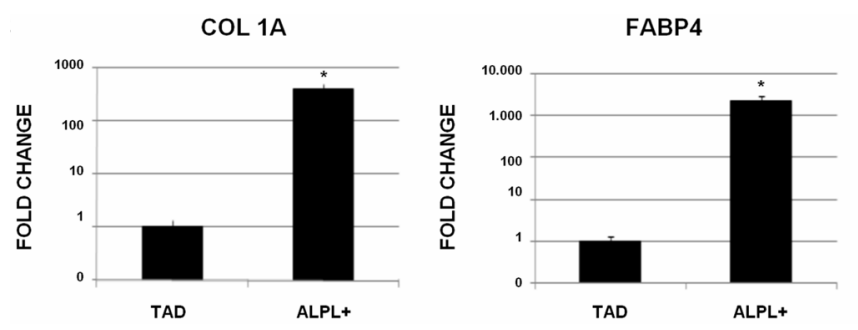

(c) studied were isolated from animal models [31,32] or from fresh material obtained during biopsies on donors with cardiac diseases [27,33]. MSC have also previously been isolated from human heart tissues taken from terminal heart failure patients undergoing heart transplantation [9]. However, heart failure may interfere with the homeostasis of the organ, thus affecting the putative cardiac progenitor population, as demonstrated by Gálvez et al. [34]. Taking these facts into account, we decided to investigate whether MSC expressing the ALPL marker could be isolated from human heart samples with no heart condition related to death cause.

In valve banks, hearts are processed for valve extraction and muscle tissue is actually discarded. We evaluated samples with a mean total ischemia time of $21.5 \pm 9.1$ hours with up to 48 hours elapsing before cell isolation.

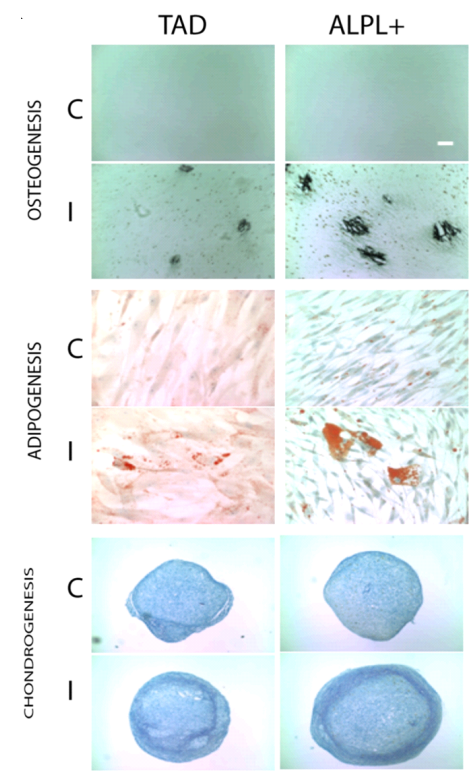

(b)

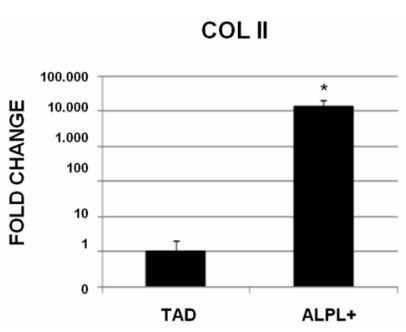

Figure 4. Differentiation into mesenchymal lineages. Differentiation into osteoblasts, adipocytes and condroblasts was induced in TAD and ALPL+ cells over a period of 21 days. Phase contrast microscopy images were obtained (a), and cytochemistry evaluations (b) carried out. Gene expression data are shown for the differentiation markers assayed by RT-qPCR (c). Data were collected from 3 donors. Representative data are shown. The calibration bar corresponds to $15 \mu \mathrm{m}$ for figure a. The calibration bar for adipogenesis in figure $b$ is $15 \mu \mathrm{m}$, and that for osteogenesis and chondrogenesis in figure $\mathrm{b}$ is $60 \mu \mathrm{m}$. Control (C) and induced (I). Fatty acid binding protein 4, adipocyte (Fabp4), collagen 1a (Col $1 \mathrm{~A})$ and collagen II (COL II), ${ }^{*} \mathrm{p}<0.05$. 


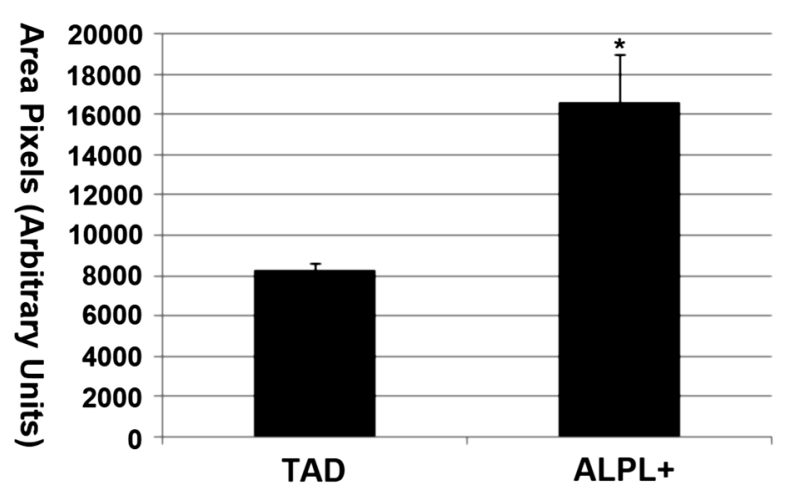

Figure 5. Quantification of the lipid-rich area during adipogenic differentiation. The area covered by lipid-rich vesicles was determined in arbitrary units (pixels). Data were collected from 3 donors ( $p<0.05$ considered significant).

We were able to isolate MSC, by the explant cell culture technique, from the right auricles of human cadaveric donors. ALPL+ cells may constitute a heart-resident population enriched in MSC, as mRNA levels for mesenchymal lineage markers (osteoblasts, adipocytes and chondroblasts) were higher in ALPL+ cells than in TAD cells induced to differentiate. Indeed, larger numbers of adipocytes were found to be differentiating cells than in TAD cells, and ALPL+ cells also had a higher lipid-rich vesicle content. Thus, the tissue discarded by tissue banks may be evaluated as a source of MSC, even after prolonged ischemia.

ALPL expression has been observed in myogenic precursors in skeletal muscle, different from satellite cells [18]. Thus, the ALPL+ cells described here may even resemble the mesoangioblasts described in murine cardiac tissue [32] and in human cardiac tissue from donors with hypertrophic cardiomyopathies [34]. ALPL+ cells may display some commitment to cardiac lineages, as we have shown they express GATA4, a master gene in cardiomyogenic and myocardial differentiation [26], the cardiac resident stem cell marker CD117 [27], ABCG2, a marker of the side population, and a well known marker of stem cells and cardiac stem cells [30], together with the cardiac isoform of troponin $\mathrm{T}$, a marker of the cardiomyocyte lineage.

The expression of these markers may constitute a suitable characteristic for evaluation, as it may be beneficial for cardiac lineage differentiation and for applications in cardiovascular cell therapy. These cells may have several advantages over other adult stem cells, because of their tissue specificity, precommitment and potential autologous use, as suggested for other cardiac progenitor cells [35]. We observed the expression of some cardiac markers in heart-derived MSC in this study. It therefore seems likely that the cardiac niche plays a role in this expression. As pointed out in a review, the stem cell niche is a

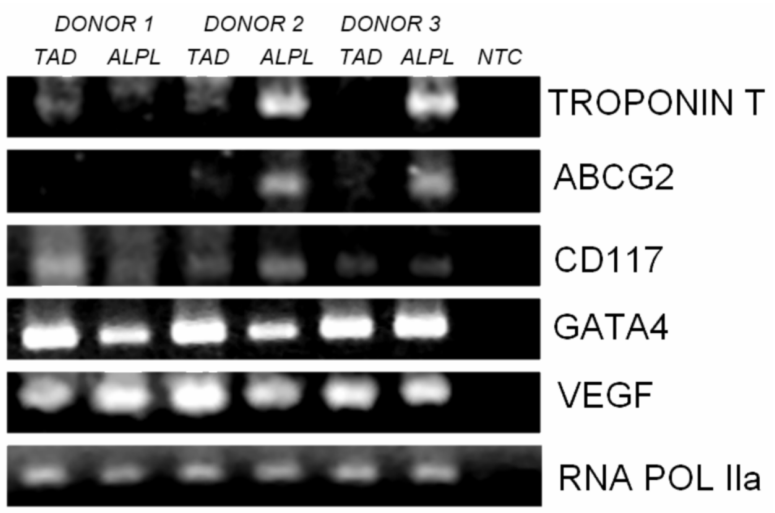

Figure 6. Expression of cardiac markers. The expression of markers for cardiac lineages, such as GATA4, CD117, ABCG2 and cardiac troponin $\mathrm{T}$, the growth factor VEGF and the housekeeping gene RNA Pol IIa was analyzed by RT-PCR for 3 independent samples of TAD cells and ALPL+ cells and a non template control (NTC). The two cell populations had similar expression profiles for VEGF, GATA4 and CD117. ALPL+ cells also expressed troponin T and ABCG2 in 2 of the 3 samples analyzed.

complex, multifactorial local micro-environment [36].

The auricle appeared to be the most appropriate source of heart-derived cells. The success rate for cell isolation by explant culture was higher for auricle-derived cells than for ventricle-derived cells. This may reflect differences in the responses to ischemia of the two types of tissue. As previously reported for experimental models, the changes induced by ischemia in atrial muscle cells occur more slower than those induced in ventricular muscle cells, and tissue autolysis is also slower $[37,38]$.

By contrast to our isolation of ALPL+ cells from human heart samples, a previous study reported an absence of ALPL detection in MSC from human hearts [39]. These conflicting findings may reflect differences in isolation and culture procedures. Here, we isolated cells by the explant cell culture technique, which may have favored the isolation of ALPL+ cells, and we used a medium described for the isolation of pericytes from human muscle tissue [18]. Cell culture enhances alkaline phosphatase expression in pericytes isolated from mammalian hearts [40]. We may therefore have favored the isolation of human heart pericytes, by selecting tissue nonspecific alkaline phosphatase-expressing cells from an explant cell culture. By contrast, in the other study, cells were obtained by enzymatic digestion of heart samples from donors with heart failure [39].

The yields of the explant culture method were compatible with successful use in basic research. However, they remained below $100 \%$, and further evaluations are required before this method can be considered for clinical use in autologous therapy. Furthermore, it takes about 
two to four weeks to isolate the first cells and, as reported here, yield varies with donor age. However, the explant cell culture method is simple, inexpensive and could be easily adapted for cell isolation from material provided by tissue banks, for obtaining heart-derived cells.

In summary, tissue and cell banks now provide hope for improvements in the quality of life of people in need of cell or tissue transplantation. In the future, allogeneic cell banks for cell therapy should be evaluated, as proposed by Pasquinelli et al. [41]. If a heart-derived cell bank could be established, this might make it easier to obtain material for cell-based therapies on the basis of a histocompatibility match. This work provides new insight into possible sources and strategies for obtaining cells and making them available for cell therapy.

\section{ACKNOWLEDGEMENTS}

We would like to thank Alyne Rocha Trigo, Andressa Vaz Schittini, Jaiesa Zych, Nilson Fidencio and Patrícia Shigunov for technical support. We also thank all the staff of Instituto Carlos Chagas-Fiocruz Paraná for laboratory and administrative support and the staff of Banco de Homoexertos Cardíacos Humanos for tissue collection. We thank Itamar Crispin for graphic design. We thank Hugo Naya from Pasteur Institute, Montevideo for statistical analysis. This study was supported by Fundação Oswaldo Cruz, PAPES V (Grant number 0232 to AC), Decit/SCTIE/MS, by CNPq and Fundação Araucária (Grant number 202/2010 to SG), SETI/Fundação Araucária (Grant number 480/2010 to AMA). AC holds a Finep/CNPq fellowship, SG and BD are research fellows at the Conselho Nacional de Desenvolvimento Ci-entifico $e$ Tecnológico-CNPq. The authors report no potential conflicts of interest or financial interests.

\section{REFERENCES}

[1] Gimble, J. and Guilak, F. (2003) Differentiation potential of adipose derived adult stem (ADAS) cells. Current Topics in Developmental Biology, 58, 137-160. doi:10.1016/S0070-2153(03)58005-X

[2] Rebelatto, C., et al. (2008) Dissimilar differentiation of mesenchymal stem cells from bone marrow, umbilical cord blood, and adipose tissue. Experimental Biology and Medicine, 233, 901-913. doi:10.3181/0712-RM-356

[3] Makino, S., et al. (1999) Cardiomyocytes can be generated from marrow stromal cells in vitro. Journal of Clinical Investigation, 103, 697-705. doi:10.1172/JCI5298

[4] Tomita, Y., et al. (2007) Application of mesenchymal stem cell-derived cardiomyocytes as bio-pacemakers: Current status and problems to be solved. Medical and Biological Engineering \& Computing, 45, 209-220. doi:10.1007/s11517-007-0163-4

[5] Toma, C., Pittenger, M., Cahill, K., Byrne, B. and Kessler, P. (2002) Human mesenchymal stem cells differentiate to a cardiomyocyte phenotype in the adult murine heart. Circulation, 105, 93-98. doi:10.1161/hc0102.101442
[6] Pittenger, M., et al. (1999) Multilineage potential of adult human mesenchymal stem cells. Science, 284, 143-147. doi:10.1126/science.284.5411.143

[7] Zuk, P., et al. (2002) Human adipose tissue is a source of multipotent stem cells. Molecular Biology of the Cell, 13, 4279-4295. doi:10.1091/mbc.E02-02-0105

[8] Rogers, I. and Casper, R. (2004) Umbilical cord blood stem cells. Best Practice and Research Clinical Obstetrics and Gynaecolog, 18, 893-908. doi:10.1016/j.bpobgyn.2004.06.004

[9] Beltrami, A., et al. (2007) Multipotent cells can be generated in vitro from several adult human organs (heart, liver, and bone marrow). Blood, 110, 3438-3446. doi:10.1182/blood-2006-11-055566

[10] Crisan, M., et al. (2008) A perivascular origin for mesenchymal stem cells in multiple human organs. Cell Stem Cell, 3, 301-313. doi:10.1016/j.stem.2008.07.003

[11] Dominici, M., et al. (2006) Minimal criteria for defining multipotent mesenchymal stromal cells. The International Society for Cellular Therapy position statement. Cytotherapy, 8, 315-317. doi:10.1080/14653240600855905

[12] Battula, V., et al. (2009) Isolation of functionally distinct mesenchymal stem cell subsets using antibodies against CD56, CD271, and mesenchymal stem cell antigen-1. Haematologica, 94, 173-184. doi:10.3324/haematol.13740

[13] Sobiesiak, M., et al. (2009) The mesenchymal stem cell antigen MSCA-1 is identical to tissue non-specific alkaline phosphatase. Stem Cells and Development, 19, 669677. doi:10.1089/scd.2009.0290

[14] Mornet, E., et al. (2001) Structural evidence for a functional role of human tissue nonspecific alkaline phosphatase in bone mineralization. Journal of Biological Chemistry, 276, 31171-31178. doi:10.1074/jbc.M102788200

[15] O'Connor, M., et al. (2008) Alkaline phosphatase-positive colony formation is a sensitive, specific, and quantitative indicator of undifferentiated human embryonic stem cells. Stem Cells, 26, 1109-1116. doi:10.1634/stemcells.2007-0801

[16] Adewumi, O. et al. (2007) Characterization of human embryonic stem cell lines by the International Stem Cell Initiative. Nature Biotechnology, 25, 803-816. doi:10.1038/nbt1318

[17] Langer, D., Ikehara, Y., Takebayashi, H., Hawkes, R. and Zimmermann, H. (2007) The ectonucleotidases alkaline phosphatase and nucleoside triphosphate diphosphohydrolase 2 are associated with subsets of progenitor cell populations in the mouse embryonic, postnatal and adult neurogenic zones. Neuroscience, 150, 863-879. doi:10.1016/j.neuroscience.2007.07.064

[18] Dellavalle, A., et al. (2007) Pericytes of human skeletal muscle are myogenic precursors distinct from satellite cells. Nature Cell Biology, 9, 255-267. doi:10.1038/ncb1542

[19] Schwab, K. and Gargett, C. (2008) Co-expression of two perivascular cell markers isolates mesenchymal stem-like cells from human endometrium. Human Reproduction, 22, 2903-2911. doi:10.1093/humrep/dem265

[20] Crisan, M., Corselli, M., Chen, C.W. and Péault, B. (2011) Multilineage stem cells in the adult: A perivascular legacy? Organogenesis, 7, 101-104. 
doi:10.4161/org.7.2.16150

[21] Chen, Z., Evans, W., Pflugfelder, S. and Li, D. (2006) Gap junction protein connexin 43 serves as a negative marker for a stem cell-containing population of human limbal epithelial cells. Stem Cells, 24, 1265-1273. doi:10.1634/stemcells.2005-0363

[22] Yoon, B., et al. (2006) Enhanced differentiation of human embryonic stem cells into cardiomyocytes by combining hanging drop culture and 5-azacytidine treatment. Differentiation, 74, 149-159. doi:10.1111/j.1432-0436.2006.00063.x

[23] Schittini, A., et al. (2010) Human cardiac explant-conditioned medium: Soluble factors and cardiomyogenic effect on mesenchymal stem cells. Experimental Biology and Medicine, 235, 1015-1024. doi:10.1258/ebm.2010.010003

[24] Shigunov, P., et al. (2011) PUMILIO-2 I is involved in the positive regulation of cellular proliferation in human adipose-derived stem cells. Stem Cells and Development, In Press. doi:10.1089/scd.2011.0143

[25] Rebelatto, C., et al. (2009) Expression of cardiac function genes in adult stem cells is increased by treatment with nitric oxide agents. Biochemical and Biophysical Research Communications, 378, 456-461. doi:10.1016/j.bbrc.2008.11.061

[26] Kelley, C., Blumberg, H., Zon, L.I. and Evans, T. (1993) GATA-4 is a novel transcription factor expressed in endocardium of the developing heart. Development, 118, 817-827.

[27] Bearzi, C., et al. (2007) Human cardiac stem cells. Proceedings of the National Academy Science of the USA, 104, 14068-14073. doi:10.1073/pnas.0706760104

[28] Formiga, F., et al. (2010) Sustained release of VEGF through PLGA microparticles improves vasculogenesis and tissue remodeling in an acute myocardial ischemia-reperfusion model. Journal of Controlied Release, 147, 30-37. doi:10.1016/j.jconrel.2010.07.097

[29] Hierlihy, A., Seale, P., Lobe, C., Rudnicki, M. and Megeney, L. (2002) The post-natal heart contains a myocardial stem cell population. FEBS Letters, 530, 239-243. doi:10.1016/S0014-5793(02)03477-4

[30] Yamahara, K., et al. (2008) Heterogeneic nature of adult cardiac side population cells. Biochemical and Biophysical Research Communications, 371, 615-620. doi:10.1016/j.bbrc.2008.04.021

[31] Beltrami, A., et al. (2003) Adult cardiac stem cells are multipotent and support myocardial regeneration. Cell, 114, 763-776. doi:10.1016/S0092-8674(03)00687-1

[32] Galvez, B., et al. (2008) Cardiac mesoangioblasts are committed, self-renewable progenitors, associated with small vessels of juvenile mouse ventricle. Cell Death and Differentiation, 15, 1417-1428. doi:10.1038/cdd.2008.75

[33] Messina, E., et al. (2004) Isolation and expansion of adult cardiac stem cells from human and murine heart. Circulation Research, 95, 911-921. doi:10.1161/01.RES.0000147315.71699.51

[34] Gálvez, B., et al. (2009) Human cardiac mesoangioblasts isolated from hypertrophic cardio-myopathies are greatly reduced in proliferation and differentiation potency. Cardiovascular Research, 83, 707-716. doi:10.1093/cvr/cvp159

[35] Bollini, S., Smart, N. and Riley, P. (2010) Resident cardiac progenitor cells: At the heart of regeneration. Journal of Molecular and Cellular Cardiology, 50, 296-303. doi:10.1016/j.yjmcc.2010.07.006

[36] Becerra, J., Santos-Ruiz, L., Andrades, J.A. and MaríBeffa, M. (2011) The stem cell niche should be a key issue for cell therapy in regenerative medicine. Stem Cell Reviews, 7, 248-255. doi:10.1007/s12015-010-9195-5

[37] Armiger, L.C., Fitzgerald, S., Vanderwee, M.A., Gavin, J.B. and Herdson, P.B. (1981) The effects of altered cation balance on the fine structure of hypoxic myocardial cells. Pathology, 13, 449-461. doi:10.3109/00313028109059063

[38] Armiger, L.C., Seelye, R.N., Morrison, M.A. and Holliss, D.G. (1984) Comparative biochemistry and fine structure of atrial and ventricular myocardium during autolysis in vitro. Basic Research in Cardiology, 79, 218-229. doi:10.1007/BF01908308

[39] Riekstina, U., et al. (2009) Embryonic stem cell marker expression pattern in human mesenchymal stem cells derived from bone marrow, adipose tissue, heart and dermis. Stem Cell Reviews, 5, 378-386. doi:10.1007/s12015-009-9094-9

[40] Lindner, H., et al. (1998) Loss of myocardial capillary endothelial-cell alkaline phosphatase (ALP) activity in primary endothelial cell culture. Cell and Tissue Research, 291, 497-505. doi:10.1007/s004410051019

[41] Pasquinelli, G., et al. (2007) Thoracic aortas from multiorgan donors are suitable for obtaining resident angiogenic mesenchymal stromal cells. Stem Cells, 25, 1627-1634. doi:10.1634/stemcells.2006-0731 